above room temperature. For lower temperatures, alcohol cooled by dry ice $\left(\mathrm{CO}_{2}\right)$ or liquid nitrogen were used to lower the temperature of the samples.

Samples tested at temperatures above $\sim 1500 \mathrm{~K}$ showed ductilities up to $7 \%$, and no work hardening. The yield stresses in that range increased with decreasing temperature. At $\sim 1500 \mathrm{~K}$ there was a transition to a brittle behavior, and the fracture stresses decreased with decreasing temperature until $\sim 1050 \mathrm{~K}$. Below $\sim 900 \mathrm{~K}$ down to room temperature, ductilities up to $8 \%$ were again observed, but the flow stress remained constant in all tests. At lower temperatures, a continuous increase in the flow stress with decreasing temperature appeared, but not as accelerated as in the tests at high temperature. The researchers observed that this behavior is similar to that of $\mathrm{Mg}_{2} \mathrm{GeO}_{4}$ olivine and some intermetallics with an $\mathrm{Ll}_{2}$ structure.

Observations of a sample tested at room temperature under transmitted polarized light showed evidence of birefringence bands. Plastically deformed samples tested below $\sim 900 \mathrm{~K}$ typically exhibit these bands. These features were not detected in samples tested above $1500 \mathrm{~K}$.

Studies about the slip systems acting in this ceramic are limited, and subsequent transmission electron microscopy (TEM) observations become necessary to determine the characteristics of the dislocation activity and its relationship with the flow process. Preliminary observations from this investigation show dislocation dipoles and stacking faults. The researchers expect to later present their results on extensive TEM observations performed in these specimens.

SIARI S. SOSA

\section{Hassium Confirmed as Group VIII Member in Periodic Table}

A team of researchers has established that element 108, hassium, forms a gaseous oxide similar to that of osmium, confirming that hassium, like osmium, is a member of Group VIII of the periodic table and should be placed directly under it. Heino Nitsche of Lawrence Berkeley National Laboratory, who is also a professor of chemistry at the University of California-Berkeley, directed the development of a very low temperature technique for separating and detecting oxides from Group VIII. The researchers used a heavy ion accelerator at the Gesellschaft für Schwerionenforschung (GSI) in Darmstadt, Germany.

Hassium does not exist in nature but must be created one atom at a time by fus- ing lighter nuclei. Energetic magnesium-26 projectiles bombarded targets of curium-248, a rare artificial isotope prepared for the experiment. Multiple curium targets were mounted in a rotating wheel system. The hassium atoms formed by impacts between target and beam reacted with oxygen to form hassium oxide molecules. The single molecules were carried through the detector by a stream of helium and immediately condensed on semiconductor diodes, arranged in rows and maintained at temperatures graded from $-20^{\circ} \mathrm{C}$ to $-170^{\circ} \mathrm{C}$.

\section{Laser Beam with Spiral Interference Pattern Allows Controlled Rotation of Micro-Objects}

By combining an annular-shaped laser beam with a reference beam, researchers from St. Andrews University in Scotland have devised a way to use optical forces to spin microscopic objects without damaging them. This system may give researchers an unprecedented amount of control for manipulating components of micromachines. So far, the scientists have demonstrated their technique with glass beads $1 \mu \mathrm{m}$ across, a 5- $\mu \mathrm{m}$-long glass rod, and a chromosome.

The technique devised by Kishan Dholakia and his colleagues relies on much of the same physics as do the wellestablished optical tweezers. In both cases, a particle gets trapped in the path of a light beam because some of the light refracts when it hits the object. This changes the momentum of the light, and, in turn, the momentum of the object, which gets attracted toward the spot in the beam where the light is most intense.

Instead of using a simple, single beam, Dholakia's group used two laser beams that combined (interfered with one another) to form a light beam with phase fronts in the form of a spiral. As reported in the May 4 issue of Science, this spiral "interference pattern" formed as the light waves that were in step with each other merged, while those out of step canceled each other out. The pattern is a spiral as the annular shaped beam had helical phase fronts. Driven by the same forces involved in optical tweezing, an object was trapped in an arm of the spiral (the brightest regions of the pattern).

The scientists then manipulated the orientation of one of the light beams, specifically changing the path length between them. This caused the spiral interference pattern between the two beams to rotate about its axis. Because the object was caught in the most intense part of the beam, it rotated as well. The researchers achieved rotation speeds over $5 \mathrm{~Hz}$. The minimum optical power needed to rotate the objects is $1 \mathrm{~mW}$.

"The beauty of our technique is that we can dictate how far we want the spiral pattern to go around and at what speed. That means we can fully control the rotation of that one particle," Dholakia said.

"Our technique could be used to drive motors, mixers, centrifuges, and other rotating parts in cheap, tiny, automated technologies of the future," he said.

\section{Noncontact Method Aligns Liquid Crystal Molecules}

Researchers at IBM have demonstrated a noncontact method that uses ion beams to align the liquid-crystal molecules inside flat-panel liquid-crystal displays (LCDs) for the display industry. Praveen Chaudhari and his research team replaced the ubiquitously used polyimide film currently used by the industry with a diamond-like carbon (DLC) film and the rubbing roller by a directed atomic beam.

As reported in the May 3 issue of Nature, the first step in this method is to deposit a thin layer of DLC (3-4-nm thick). Next, low energy atoms are shot by an ion gun at an angle to the surface of the substrate. This introduces orientational order by a selective process in which the rings of the DLC film that are perpendicular to the ion beam are preferentially destroyed compared to those parallel. The net result is an excess amount of rings whose planes are parallel to the direction of the ion beam. These rings then produce alignment of the rod-shaped liquid crystals.

This noncontact method eliminates many problems associated with the current rubbing method. Rubbed displays must be removed from the clean room manufacturing line to avoid contamination from the velvet cloths used in rubbing. Then they must be washed and baked to eliminate contaminants before returning to the manufacturing line. Furthermore, worn velvet cloths are replaced periodically, interrupting the manufacturing process. Defects caused by the rubbing process cannot be detected until much later in the manufacturing process, when they are difficult or impossible to correct.

The noncontact method is built into the clean manufacturing line, eliminating those extra steps. A noncontact alignment process has been the goal of liquid-crystal display technologists for over two decades since the high-resolution displays were envisioned. 